\title{
Experimentally validated analytical expressions for the thermal efficiencies and thermal resistances of porous metal foam-fins
}

\author{
J.R. Stark ${ }^{\mathrm{a}^{*}}$ \\ R. Prasad ${ }^{\mathrm{a}}$ \\ T.L. Bergman ${ }^{\mathrm{a}}$
}

${ }^{a}$ Department of Mechanical Engineering, The University of Kansas, 1530 W. $15^{\text {th }}$ Street 3138, Lawrence, KS 66045, USA

"Corresponding Author

John R. Stark

John.Robert.Stark@gmail.com

Ph: +1 5152914101 


\begin{abstract}
Novel, analytical expressions are developed for the thermal efficiency and thermal resistance associated with forced convection and conduction within a cylindrically shaped, open porous medium that is attached concentrically to a circular tube. Using the new analytical expression in conjunction with a two-temperature porous medium numerical model, a companion semi-analytical expression for the thermal efficiency of a cubical foam block attached on-center to a circular tube is also developed. Heat rates predicted with the semi-analytical model are verified through comparison with experimentally measured heat transfer rates using open-cell metal foam. Foam thermal resistances are also quantified over a range of operating conditions. The recommended ranges of application of the analytical and experimentally verified semianalytical expressions for the thermal efficiency of both annular and square-shaped foams are also presented.
\end{abstract}

\title{
Keywords
}

Thermal Efficiency; Metal Foam; Porous Media Convection; Numerical Modeling; Analytical Modeling 


\section{Nomenclature}

\begin{tabular}{|c|c|}
\hline$A$ & $\operatorname{area}\left(\mathrm{m}^{2}\right)$ \\
\hline$r^{\prime}, \tilde{r}$ & geometric constants \\
\hline$c_{p}$ & specific heat at constant pressure $(\mathrm{J} / \mathrm{kg} \mathrm{K})$ \\
\hline$D$ & diameter, unit cell length (m) \\
\hline$h$ & convection coefficient $\left(\mathrm{W} / \mathrm{m}^{2} \mathrm{~K}\right)$ \\
\hline$H$ & height $(\mathrm{m})$ \\
\hline$k$ & thermal conductivity $(\mathrm{W} / \mathrm{m} \mathrm{K})$ \\
\hline$K$ & permeability $\left(\mathrm{m}^{2}\right)$ \\
\hline$K_{\text {loss }}$ & loss coefficient $\left(\mathrm{m}^{-1}\right)$ \\
\hline$L$ & length (m) \\
\hline LMTD & log mean temperature difference $\left({ }^{\circ} \mathrm{C}\right)$ \\
\hline$m$ & porous matrix constant $\left(\mathrm{m}^{-1}\right)$ \\
\hline$\dot{m}$ & mass flow rate $(\mathrm{kg} / \mathrm{s})$ \\
\hline $\mathrm{Nu}$ & Nusselt number \\
\hline$p$ & pressure $(\mathrm{Pa})$ \\
\hline $\operatorname{Pr}$ & Prandtl number \\
\hline$q$ & heat rate $(\mathrm{W})$ \\
\hline$q^{\prime}$ & heat rate per unit length $(\mathrm{W} / \mathrm{m})$ \\
\hline$r$ & radius $(\mathrm{m})$ \\
\hline$r_{1}, r_{2}$ & inner radius, outer radius $(\mathrm{m})$ \\
\hline$R$ & thermal resistance $(\mathrm{K} / \mathrm{W})$ \\
\hline$R^{\prime}$ & length based thermal resistance (m K/W) \\
\hline$R^{\prime \prime}$ & area based thermal resistance $\left(\mathrm{m}^{2} \mathrm{~K} / \mathrm{W}\right)$ \\
\hline $\operatorname{Re}$ & Reynolds number \\
\hline$T$ & temperature $\left({ }^{\circ} \mathrm{C}\right)$ \\
\hline$t$ & thickness (m) \\
\hline$u$ & velocity $(\mathrm{m} / \mathrm{s})$ \\
\hline$V$ & volume $\left(\mathrm{m}^{3}\right)$ \\
\hline$W$ & width (m) \\
\hline$x, y, z$ & coordinate directions \\
\hline \multicolumn{2}{|l|}{ Greek } \\
\hline$\alpha_{v}$ & surface area per unit volume $\left(\mathrm{m}^{2} / \mathrm{m}^{3}\right)$ \\
\hline$\varepsilon$ & porosity \\
\hline$\eta$ & porous matrix efficiency \\
\hline$\theta$ & excess temperature $\left({ }^{\circ} \mathrm{C}\right)$ \\
\hline$\mu$ & dynamic viscosity (Pa s) \\
\hline$\rho$ & density $\left(\mathrm{kg} / \mathrm{m}^{3}\right)$ \\
\hline
\end{tabular}




$\begin{array}{ll}\begin{array}{ll}\text { Superscripts } \\ -\end{array} & \\ & \text { average } \\ \text { Subscripts } & \\ 0,1 & \text { Bessel series order } \\ a & \text { annular } \\ b & \text { base, bottom } \\ c & \text { characteristic, condenser, contact, cross-section } \\ \text { ch } & \text { channel } \\ \text { cond } & \text { conduction } \\ \text { conv } & \text { convection } \\ \text { eff } & \text { effective } \\ f & \text { fiber, fluid, foam } \\ \text { hp } & \text { heat pipe } \\ \text { hs } & \text { heat spreader } \\ \text { i } & \text { insulation } \\ \text { in } & \text { insert } \\ l m & \text { logarithmic mean } \\ o & \text { outlet } \\ p & \text { pore } \\ r & \text { radial } \\ s & \text { solid, surface } \\ t & \text { top } \\ \text { tc } & \text { thermocouple } \\ \text { tp } & \text { thermal paste } \\ s q & \text { square } \\ \text { tot } & \text { total } \\ \infty & \text { ambient } \\ & \end{array}$

\section{Introduction}

Owing to their extremely large surface areas per unit volume, open-cell high-porosity metal foams have many potential heat transfer applications [1-3]. Heat transfer within a metal foam (depicted in Fig. 1) is complex, consisting of conduction within the metal component, convection between the metal and the saturating working fluid, and potentially radiation 
throughout the fluid domain. Numerous efforts have been made to model the heat transfer processes using both multi-phase local, and continuum approaches. Local multi-phase analyses are often performed with the intent of informing continuum scale models through the quantification of effective thermal transport properties.

Extensive work has been carried out to generate an idealized solid phase structure for a typical unit cell within the foam, providing the geometrical information needed to develop the multi-phase local models and effective properties. The approximation of a general, disordered porous medium by using idealized unit cells was shown by Koch et al. [4] to be valid in ultimately predicting effective transport properties. Analytical modeling of the conduction and convection heat transfer within a linear cylinder lattice unit cell (characterized by a grid of uniform diameter cylinders that meet orthogonally) was first proposed by Lu et al. [5], who used cylinder-in-cross flow correlations to approximate the convective heat transfer processes. Du Plessis et al. [6] performed the first numerical analysis of the three-dimensional steady flow through a simple unit cell.

Conduction and convection within a more geometrically complex unit cell, which was first proposed by Weaire and Phalen [7], was numerically simulated by Boomsma et al. [8], Kopanidis et al. [9], and others, yielding reasonable predictions of experimentally measured pressure drops associated with flow through the foam structure. Krishman et al. [10] used a similar approach with a less complex Kelvin unit cell [11] while Iasiello et al. [12] compared predicted heat transfer rates and pressure drops associated with the Weaire-Phalen and Kelvin unit cells, showing that the more simplified Kelvin model is acceptable for the prediction of areaaveraged convection coefficients and overall pressure loss characteristics. Numerical modeling of convection and conduction by Ranut et al. [13] utilized an experimentally determined foam 
geometry, with predictions showing good agreement with those associated with previous unit cell modeling efforts.

Continuum-based, analytical modeling of heat transfer within highly porous metal foams has also been conducted. Using a two-temperature (solid-fluid) approach, an analytical description of one-dimensional Cartesian conduction and convection within a porous medium was developed by Lee and Vafai [14]. Similar modeling of forced convection and conduction within open-cell, high-porosity metal foams has been achieved and described in terms of a thermal efficiency [15-20] similar to the traditional efficiency of thin solid fins [21]. In a related study, heat transfer within annular porous media experiencing forced convection within a concentric tube heat exchanger that is filled with aluminum foam was analytically described by Zhao et al. [22]. Experimental and numerical investigations of a cylinder with an attached annular foam block have been performed by T'Joen et al. [23] and Odabaee et al. [24], respectively.

In this study, $(i)$ a continuum-based, analytical description for conduction and forced convection heat transfer within an annular porous matrix attached concentrically to a circular tube, reported in terms of a thermal efficiency, will be developed, (ii) a continuum-based numerical model will be used to develop a semi-analytical expression for the thermal efficiency of a cubical porous block attached on-center to a circular tube, and (iii) the thermal behavior predicted with the semi-empirical expression will be compared to experimental measurements conducted with convectively cooled foam blocks of square cross section attached on-center to a nearly isothermal warm cylinder (heat pipe) using a unique experimental setup.

\section{Analytical continuum model for annular, foam-based extended surfaces}


Consider a cool, ambient fluid that is forced through an open-cell, annular metal foam block that is attached to the exterior of a hot cylindrical tube (or heat pipe), as illustrated in Fig. 2(a). Consistent with standard fin analyses [21], steady, one-dimensional conduction heat transfer in the radial direction is assumed. In addition, the block is taken to be isotropic with constant properties, and both (i) radiation heat transfer and (ii) heat transfer in the radial direction at $r=r_{2}$ are assumed to be negligible. The fluid flow within the porous material is assumed to provide a uniform convection coefficient between the fluid and solid phase, and is described by a characteristic temperature, that is either (i) that of the ambient fluid (as in the analysis below) or (ii) an appropriate log-mean temperature (as in a the comparison of model predictions with experimental results to be discussed later). Conduction within the gas can be shown to be negligible for the porous medium and fluid of interest here, and is neglected.

\subsection{Analysis}

Applying conservation of thermal energy to a radial differential element within the porous cylindrical block, as illustrated in Fig. 2(b), yields

$$
\frac{d^{2} T}{d r^{2}}+\left(\frac{1}{A_{c, r}} \frac{d A_{c, r}}{d r}\right) \frac{d T}{d r}-\left(\frac{1}{A_{c, r}} \frac{\bar{h}}{k_{s, \text { eff }}} \frac{d A_{s}}{d r}\right)\left(T-T_{\infty}\right)=0
$$

where the first and second terms are associated with the radial conduction through the solid phase of the metal foam, and the third term represents the convection heat transfer from the solid phase to the fluid. The area normal to conduction in the radial direction and the differential surface area experiencing convection are

$$
A_{c, r}=2 \pi r t \quad \text { and } \quad d A_{s}=\alpha_{v} d V=\alpha_{v} d\left[t\left(r^{2}-r_{1}^{2}\right) \pi\right]
$$


respectively. Here, $\alpha_{v}$ is the specific surface area (surface area per unit volume) of the porous medium and $k_{s, \text { eff }}$ is the effective thermal conductivity of the solid phase. The heat transfer coefficient within the medium, $\bar{h}$, represents the spatially averaged value of the local heat transfer coefficient, $h$, at the metal-fluid interfaces. Defining an excess temperature, $\theta(r) \equiv T(r)-T_{\infty}$, Eq. (1) may be simplified to

$$
\frac{d^{2} \theta}{d r^{2}}+\frac{1}{r} \frac{d \theta}{d r}-m^{2} \theta=0 \quad \text { where } \quad m^{2}=\frac{\bar{h} \alpha_{v}}{k_{s, \text { eff }}}
$$

When solved under the boundary conditions of $(i)$ constant base temperature, $\theta\left(r_{1}\right)=\theta_{b}$, and (ii) adiabatic behavior at the outer radius of the annular block, $\left.(d \theta / d r)\right|_{r_{2}}=0$, expressions for the annular porous matrix heat rate and porous matrix efficiency for the annular block, $\eta_{a} \equiv q\left(r_{1}\right) /\left[\bar{h} \alpha_{v} t\left[\left(r_{2}^{2}-r_{1}^{2}\right) \theta_{b}\right]\right.$ may be written as

$$
q\left(r_{1}\right)=2 \pi k_{s, \text { eff }} r_{1} t \theta_{b} m \frac{K_{1}\left(m r_{1}\right) I_{1}\left(m r_{2}\right)-I_{1}\left(m r_{1}\right) K_{1}\left(m r_{2}\right)}{K_{0}\left(m r_{1}\right) I_{1}\left(m r_{2}\right)+I_{0}\left(m r_{1}\right) K_{1}\left(m r_{2}\right)}
$$

and

$$
\eta_{a}=\frac{\bar{\theta}}{\theta_{b}}=\frac{2 r_{1}}{m\left(r_{2}^{2}-r_{1}^{2}\right)}\left(\frac{K_{1}\left(m r_{1}\right) I_{1}\left(m r_{2}\right)-I_{1}\left(m r_{1}\right) K_{1}\left(m r_{2}\right)}{K_{0}\left(m r_{1}\right) I_{1}\left(m r_{2}\right)+I_{0}\left(m r_{1}\right) K_{1}\left(m r_{2}\right)}\right)
$$

respectively. Note that the metal surface area at $r=r_{2}$ is small compared to the surface area within the porous array, justifying the use of the adiabatic tip condition. The porous matrix efficiency, $\eta_{a}$, is analogous to the traditional fin efficiency [21] and leads to an expression for the equivalent thermal resistance associated with the combined conduction and convection processes within the foam, which may be defined as

$$
R_{f, a}=\frac{\theta_{b}}{q\left(r_{1}\right)}=\frac{1}{A_{s} \eta_{a} \bar{h}}=\frac{1}{\alpha_{v} t \pi\left(r_{2}^{2}-r_{1}^{2}\right) \eta_{a} \bar{h}}
$$


The preceding thermal resistance may be utilized in resistance-based analyses of complex thermal systems such as those described in [21, 25-27] In addition, a resistance per unit cylinder length may be defined as

$$
R_{f, a}^{\prime}=\frac{\theta_{b}}{q^{\prime}\left(r_{1}\right)}=\frac{t}{A_{s} \eta_{a} \bar{h}}=\frac{1}{\alpha_{v}\left(r_{2}^{2}-r_{1}^{2}\right) \pi \eta_{a} \bar{h}}
$$

\subsection{Determination of $\alpha_{v}, k_{s, \mathrm{eff}}$, and $\bar{h}$}

Implementation of the preceding analysis necessitates quantification of the relevant geometric and transport terms, $\alpha_{v}, k_{s, \text { eff }}$, and $\bar{h}$. The values of the terms may be specified, or calculated using appropriate sub-models for specific types of foams and operating conditions. The porous medium used to demonstrate the application of the analytical solutions and in the experiments of Section 4 is an open-cell, aluminum foam (Duocel $\left.{ }^{\circledR} \mathrm{Al} 10-44\right)$ characterized by a measured porosity of $\varepsilon=0.941$ and a nominal pore size of 10 pores per inch (PPI). The methodology of Calmidi [28] is used to determine a characteristic pore size, $D_{p}$, based upon the pore density (expressed in PPI or pores per meter, PPM) and the porosity, $\varepsilon$, as in Eq. (8a) [28]. The characteristic metal fiber diameter of the foam, $D_{f}$, is then the difference between the average unit cell length, $D$ (that includes both pores and fibers), and $D_{p}$ as expressed in Eq. (8b) [28].

$$
D_{p}=\frac{1}{P P I(\text { or } P P M)}\left(1-2 \sqrt{\frac{(1-\varepsilon)}{3 \pi}}\right) ; D_{f}=D-D_{p}
$$

The specific surface area is then determined from the values of $D_{f}$ and $D_{p}[28]$ as

$$
\alpha_{v}=\frac{3 \pi D_{f}}{D_{p}^{2}}
$$


Many models for the effective thermal conductivity of aluminum foams have been

reported, as recently reviewed by Wang and Li [29]. To be consistent with Eq. (1), an

analytically derived correlation for the effective thermal conductivity within only the solid phase, $k_{s, \text { eff }}$ for Duocel® aluminum foams [30] is utilized

$$
k_{s, \mathrm{eff}}=\left[\frac{2}{\sqrt{3}}\left(\frac{r^{\prime} \tilde{r}}{(1+\tilde{r}) k_{s}}+\frac{\left(1-r^{\prime}\right) \tilde{r}}{\frac{2}{3} \tilde{r} k_{s}}+\frac{\frac{\sqrt{3}}{2}-\tilde{r}}{\frac{4 r^{\prime}}{3 \sqrt{3}} \tilde{r} k_{s}}\right)\right]^{-1}
$$

where $r^{\prime}=0.09$ and $\tilde{r}$ is a geometric parameter expressed as

$$
\tilde{r}=\frac{-r^{\prime}+\sqrt{r^{\prime 2}+4(1-\varepsilon) \frac{\sqrt{3}}{6}\left[2-r^{\prime}\left(1+\frac{4}{\sqrt{3}}\right)\right]}}{\frac{2}{3}\left[2-r^{\prime}\left(1+\frac{4}{\sqrt{3}}\right)\right]}
$$

Local (equal to area-averaged) heat transfer coefficients are calculated via an experimentally derived correlation provided by Park et al. [18], where the Nusselt and Reynolds numbers are based upon the metal fiber's characteristic diameter, $D_{f}$, and the Reynolds number, $R e_{D_{f}}=u_{p} D_{f} / v_{f}$, is a function of the average pore velocity $\left(u_{p}=u_{\infty} / \varepsilon\right)$

$$
N u_{D_{f}}=\frac{\bar{h} D_{f}}{k_{f}}=0.039 \operatorname{Re}_{D_{f}}^{0.8} \operatorname{Pr}^{0.4}
$$

\section{Numerical model}

Porous metal foams are commercially available in rectangular block forms with a square footprint, suggesting the relevance and importance of extending the preceding analysis for the 
annular block to square geometries. To do so, a series of numerical simulations involving either square or annular blocks is performed using a consistent set of assumptions as described for the preceding analysis, with the exception that the numerical simulations include three-dimensional effects. The predicted efficiencies associated with the two foam geometries of Fig. 3 are then compared over a range of square block lengths $\left(L_{s q}\right)$, annular block radius ratios $\left(r_{2} / r_{1}\right)$, and porous matrix constants $(m)$ as defined in Eq. (3), in order to describe the square block behavior with expressions similar to Eq. (5).

The gas within the porous medium is assumed to be incompressible. Symmetry is taken advantage of to reduce computational expense, resulting in the $45^{\circ}$ computational domains for the (i) annular and (ii) square block cases of Fig. 3. (The annular and square block domains of Fig. 3 are considered independently in the simulations. The $45^{\circ}$ square domain represents the smallest possible for that geometry. The $45^{\circ}$ annular domain was chosen to match the square domain.) Both geometries are described in terms of a common outer lateral dimension of $r_{2}=$ $L_{s q} / 2$ and common thickness, $t$. Heat transfer within each domain is governed by a twotemperature porous model [31], which describes both the moving fluid (air) within the porous medium, and the solid component of the medium (aluminum). Heat transfer and fluid flow within the air are governed by conservation of energy, Eq. (13), conservation of mass, Eq. (14), and conservation of momentum, Eqs. $(15 \mathrm{a}-15 \mathrm{c})$. Momentum conservation is described by the Brinkman-Forchheimer-extended Darcy model [31, 32], which includes both pressure loss terms associated with the Darcy coefficient $(K)$ and second order losses ( $\left.K_{\text {loss }}\right)$, as calculated using the correlations provided by Calmidi [28].

$$
\varepsilon \rho_{f} c_{p, f}\left(u_{x} \frac{\partial T_{f}}{\partial x}+u_{y} \frac{\partial T_{f}}{\partial y}+u_{z} \frac{\partial T_{f}}{\partial z}\right)=k_{f, \text { eff }}\left(\frac{\partial^{2} T_{f}}{\partial x^{2}}+\frac{\partial^{2} T_{f}}{\partial y^{2}}+\frac{\partial^{2} T_{f}}{\partial z^{2}}\right)+q_{s f}
$$




$$
\begin{aligned}
& \frac{\partial u_{x}}{\partial x}+\frac{\partial u_{y}}{\partial y}+\frac{\partial u_{z}}{\partial z}=0 \\
& \frac{\rho_{f}}{\varepsilon}\left(u_{x} \frac{\partial u_{x}}{\partial x}+u_{y} \frac{\partial u_{x}}{\partial y}+u_{z} \frac{\partial u_{x}}{\partial z}\right)=-\frac{\partial p}{\partial x}+\frac{\mu}{\varepsilon}\left(\frac{\partial^{2} u_{x}}{\partial x^{2}}+\frac{\partial^{2} u_{x}}{\partial y^{2}}+\frac{\partial^{2} u_{x}}{\partial z^{2}}\right)-\frac{\mu}{K} u_{x}-\varepsilon K_{\text {loss }} \frac{\rho_{f} \varepsilon}{2}|u| u_{x} \\
& \frac{\rho_{f}}{\varepsilon}\left(u_{x} \frac{\partial u_{y}}{\partial x}+u_{y} \frac{\partial u_{y}}{\partial y}+u_{z} \frac{\partial u_{y}}{\partial z}\right)=-\frac{\partial p}{\partial y}+\frac{\mu}{\varepsilon}\left(\frac{\partial^{2} u_{y}}{\partial x^{2}}+\frac{\partial^{2} u_{y}}{\partial y^{2}}+\frac{\partial^{2} u_{y}}{\partial z^{2}}\right)-\frac{\mu}{K} u_{y}-K_{\text {loss }} \frac{\rho_{f} \varepsilon}{2}|u| u_{y} \\
& \frac{\rho_{f}}{\varepsilon}\left(u_{x} \frac{\partial u_{z}}{\partial x}+u_{y} \frac{\partial u_{z}}{\partial y}+u_{z} \frac{\partial u_{z}}{\partial z}\right)=-\frac{\partial p}{\partial z}+\frac{\mu}{\varepsilon}\left(\frac{\partial^{2} u_{z}}{\partial x^{2}}+\frac{\partial^{2} u_{z}}{\partial y^{2}}+\frac{\partial^{2} u_{z}}{\partial z^{2}}\right)-\frac{\mu}{K} u_{z}-K_{\text {loss }} \frac{\rho_{f} \varepsilon}{2}|u| u_{z}
\end{aligned}
$$

Heat transfer in the solid domain is governed by conservation of energy

$$
0=k_{s, \mathrm{eff}}\left(\frac{\partial^{2} T_{s}}{\partial x^{2}}+\frac{\partial^{2} T_{s}}{\partial y^{2}}+\frac{\partial^{2} T_{s}}{\partial z^{2}}\right)+q_{f s}
$$

where the heat transferred between the two media is represented by

$$
q_{s f}=-q_{f s}=\bar{h} \alpha_{v}\left(T_{s}-T_{f}\right)
$$

The convection coefficient is calculated from Eq. (12) and the specific surface area (associated with the exposed surface area of the solid, shown in Fig. 1) is calculated from Eq. (9).

Both the solid and gas domains are subject to a common base temperature of $T_{b}$ at $r_{1}$. Adiabatic conditions are applied to both the solid and the gas at the outer edges of the two blocks in order to be consistent with the annular domain analysis, while symmetry conditions are assumed along the thin, vertical faces at $\theta=0^{\circ}$ and $45^{\circ}\left(45^{\circ}\right.$ and $\left.90^{\circ}\right)$ for the annular (square) domain of Fig. 3. Adiabatic boundary conditions are applied to the solid at the top and bottom surfaces $(z= \pm t / 2)$ of the domain. The gas enters the domain normal to the top surface $(z=+t / 2)$ at a specified ambient temperature and the gas exits the $z=-t / 2$ surface at zero relative pressure and with a zero second spatial derivative of the fluid temperature in the $z$-direction. No-shear 
velocity conditions are applied at $r_{1}$ and $r_{2}\left(L_{s q} / 2\right)$ for the annular (square) cases in an effort to maintain uniform flow in the numerical predictions to be consistent with the preceding analysis.

The computational mesh, which was assessed for grid-independence as described in the Appendix, consists of approximately 100,000 elements. Convergence is assumed once all RMS values of all dependent variables are below $10^{-7}$. The results reported here entail a total of 476 simulations performed using Ansys CFX (17.1) on a Dell Precision T7600.

\section{Experiments}

Experiments were conducted to both validate and demonstrate usage of analytical expressions for the efficiency of a square porous matrix, to be developed in Section 5.2.

\subsection{Experimental apparatus}

A flow channel similar to the one described in Stark et al. [33] was used to generate benchmark data. An electrically heated heat pipe (HP) of cylindrical cross section is used to deliver thermal energy to the bases (at $r=r_{1}$ ) of four, stacked square porous block structures which surround the condenser section of the HP. The heat is ultimately transferred to cold air drawn through the channel and porous metal blocks. The channel is similar to (but smaller than) that of Fig. 4 of [33] with a length $L_{c h}=1.3 \mathrm{~m}$ and cross sectional width and height of $W_{c h}=100$ $\mathrm{mm}$ and $H_{c h}=120 \mathrm{~mm}$, respectively.

A copper- $\mathrm{H}_{2} \mathrm{O} \mathrm{HP}$ with a sintered copper wick (Enertron HP-HD06DI17500BA) is positioned vertically in a HP carrier that can be snapped into place through the bottom of the channel, as shown in Fig. 3(a). The HP is of diameter $D_{h p}=6 \mathrm{~mm}$ and length $L_{h p}=175 \mathrm{~mm}$; additional geometric dimensions as well as physical and effective properties are reported 
elsewhere [33-35]. Within the heavily insulated carrier is a cylindrical aluminum heat spreader $\left(\mathrm{Al} 2024-\mathrm{T} 4 ; k_{h s}=121 \mathrm{~W} / \mathrm{m} \cdot \mathrm{K}\right)$ which surrounds the evaporator section of the $\mathrm{HP}$ of length $L_{e}=$ $50.8 \mathrm{~mm}$. The spreader has an inner (outer) diameter of $6.05 \mathrm{~mm}(25.4 \mathrm{~mm})$ and a height of $L_{h s}=$ $L_{e}$. The $0.05 \mathrm{~mm}$ gap between the HP and heat spreader is packed with thermal paste (Artic Silver $\left.5 ; k_{t p}=8.7 \mathrm{~W} / \mathrm{m} \mathrm{K}\right)$. Thermal energy is provided to the exterior of the heat spreader by an adhesive-backed electrical patch heater (McMaster 35765K634) [33] energized by a DC power supply (Dr. Meter HY3005F-3) and is transferred radially inward to the HP. The power dissipation (heat rate, $q$ ) is measured by an ammeter (FLUKE 77 IV; accuracy $\pm 1.5 \%$ of reading +2 LSD; resolution $1 \mathrm{~mA}$ ) and a voltmeter (AMPROBE AM-510; accuracy $\pm 0.8 \%$ of reading +1 LSD; resolution $10 \mathrm{mV}$ ).

The condenser section of the HP is of length $L_{c}=51 \mathrm{~mm}$ and is placed at the centers of four, stacked blocks of high porosity aluminum foam (10 PPI Duocel® Al 10-44), each block with an outer dimension of $L_{s q}=100 \mathrm{~mm}$ and thickness $12.7 \mathrm{~mm}$, as shown in Fig. 3(b). The blocks have a measured average porosity of $\varepsilon=0.941$. Each foam block is attached to a threaded aluminum insert fabricated of the same alloy as the heat spreader previously described. The solid inserts have inner diameters of $6.05 \mathrm{~mm}$ and outer threaded diameters of $25.4 \mathrm{~mm}$ with UNC 1-8 threads [36]. To minimize the thermal contact resistance between the foam blocks and the threaded solid inserts, an undersized hole (diameter $5 \mathrm{~mm}$ ) is first drilled through the center of the foam's largest face, then the insert is screwed into the foam block, crushing the aluminum foam into the screw threads. The $0.05 \mathrm{~mm}$ gaps between the inserts and the HP are also packed with Artic Silver thermal paste. Closed-cell styrofoam blocks $\left(100 \mathrm{~mm} \times 100 \mathrm{~mm}, L_{i, t}=55 \mathrm{~mm}\right.$ and $L_{i, b}=14.2 \mathrm{~mm}, k_{i}=0.033 \mathrm{~W} / \mathrm{m} \mathrm{K}$ ) are installed above and below the metal foam blocks to reduce heat losses and ensure that the channel air is directed entirely through the foam matrices. 
Detailed information regarding measurement of local temperatures, local and average channel air velocities, as well as the calibration and validation procedures may be found in [33].

\subsection{Analysis of Experimental Measurements}

The experiments are used to quantify the thermal resistance of the foam block, $R_{f}$, and to test a new expression for the thermal efficiency of the square foam block that is developed in

\section{Section 5.2.}

Heat is transferred from the electric patch heater to the air within the foam (at either $T_{\infty}$ or $\left.T_{l m}\right)$ through the thermal resistance network of Fig. 3(c). A local temperature within the heat spreader, of value $T_{t c}$, is measured at a known radial location as shown in Fig. 3(a). The conduction resistance between the location associated with $T_{c}$ and the inner wall of the heat spreader is calculated to be $R_{h s}=0.019 \mathrm{~K} / \mathrm{W}$ using a one-dimensional conduction analysis [21]. Heat is conducted through the thermal paste $\left(R_{t p, e}=0.003 \mathrm{~K} / \mathrm{W}\right)$ to the exterior of the HP evaporator section at temperature $\bar{T}_{e}$. The complex, multi-phase conduction, convection, and phase change heat transfer mechanisms within the HP walls, wick and working fluid are modeled using the methodology of Sharifi et al. [35], who ultimately reported a thermal resistance of $R_{h p}$ $\approx 0.22 \mathrm{~K} / \mathrm{W}$ for the HP used here operating under similar evaporator and condenser wall temperatures. Since the HP evaporator and condenser sections are of equal length, $R_{t p, c}=R_{t p, e}$ while the radial conduction resistance associated with the aluminum inserts is $R_{i n, r}=0.037 \mathrm{~K} / \mathrm{W}$ based on a one-dimensional conduction analysis and assuming an outer insert radius located midway between the crown and root of the threading. The contact resistance per unit area between the highly porous metal foam and the threaded aluminum insert is estimated to be $R_{t, c}^{\prime \prime}$ $=0.0246 \mathrm{~K} \cdot \mathrm{m}^{2} / \mathrm{W}$ and is taken from the experimentally derived values reported by Sadeghi et al. 
[37] for the lowest contact pressure (0.5 MPa), which is applied to the total area of the threading. This yields a thermal resistance of $R_{t, c}=0.38 \mathrm{~K} / \mathrm{W}$. Note that parasitic heat losses were measured by removing the heat pipe/foam assembly, and were found to be small (approximately 5 percent of the electrical power). The parasitic losses were subtracted from the measured electrical power and the corrected heat transfer rates are used in Section 5.3.

The sum of the thermal resistances between the base of the foam and the location corresponding to $T_{t c}$ is equal to $0.71 \mathrm{~K} / \mathrm{W}$. The lone remaining thermal resistance in the network, $R_{f}$, is associated with the conjugate heat transfer within the square metal foam. Its value will be determined via a new, semi-analytical expression for the efficiency of the square metal block, to be developed in Section 5.2.

\section{Results}

\subsection{Analytical results and numerical predictions}

Validation of the numerical model. Numerically predicted porous matrix efficiencies for the annular porous block, as calculated by Eq. (18), are first compared to the analytically derived efficiency provided in Eq. (5).

$$
\eta_{a} \equiv \frac{\int_{V} \bar{h} \alpha_{v}\left[T(x, y, z)-T_{\infty}\right] d V}{\bar{h} \alpha_{v}\left(T_{b}-T_{\infty}\right) V}
$$

For this comparison exercise, an annular block of thickness $t=1 \mathrm{~mm}$, outer radius $r_{2}=50 \mathrm{~mm}$, and various inner radii, $r_{1}$, are specified. The base and ambient temperatures are set to $T_{b}=60^{\circ} \mathrm{C}$ and $T_{\infty}=25^{\circ} \mathrm{C}$, respectively. The relatively small thickness of the computational domain, in conjunction with a relatively high ambient velocity, $u_{\infty}=1 \mathrm{~m} / \mathrm{s}$, limits the numerically predicted temperature rise of the air as it passes through the medium to $\Delta T \lesssim 0.3^{\circ} \mathrm{C}$, consistent with the 
assumption of the gas temperature being at its ambient value in the development of Eq. (5). To achieve consistent values of $m L_{c}$ for a range of inner radii (where $L_{c}=r_{2}-r_{1}$ ) convection heat transfer coefficients $(\bar{h})$, specific areas $\left(\alpha_{v}\right)$, and effective solid phase thermal conductivities

$\left(k_{s, \text { eff }}\right)$ were parametrically varied. Specifically, predictions were generated over the ranges 0.001 $\leq m L_{c} \leq 2.5$ and $1.2 \leq r_{2} / r_{1} \leq 10$. Gas properties are evaluated at $p=1$ atm and $T=25^{\circ} \mathrm{C}$, while $k_{s}$ $=218 \mathrm{~W} / \mathrm{m} \cdot \mathrm{K}[38]$ yielding $k_{s, \text { eff }}=4.62 \mathrm{~W} / \mathrm{m} \cdot \mathrm{K}$.

Analytically determined (lines) and numerically predicted values (circles) of the porous matrix efficiencies for the annular block are shown in Fig. 5(a). The efficiency decreases as either $m L_{c}$ or $r_{2} / r_{1}$ increases, in a manner similar to the efficiency behavior of solid, annular fins [21]. Over the entire ranges of $m L_{c}$ and $r_{2} / r_{1}$ used to generate Fig. 5(a), the mean difference between the analytically determined and numerically predicted efficiencies is less than $0.05 \%$. Hence, the numerical model is deemed to be validated.

Numerically predicted efficiencies of annular and square foam blocks. Representative numerically predicted porous matrix efficiencies for annular and square blocks are shown in Figs. 5(b) through 5(d). The predictions were generated using the same $m L_{c}$ and $r_{2} / r_{1}$ ranges as in Fig. 5(a), but with $r_{2}$ replaced by $L_{s q} / 2$ when square blocks are considered. As evident, the annular block efficiencies are consistently higher than those of the square blocks at any given $m L_{c}$ value. This is expected due to the relatively low local heat fluxes in the corner regions of the square block, reducing their efficiency relative to that of the annular blocks.

\subsection{Mathematical expressions for the efficiency of square foam blocks}


The main objective of this investigation is to develop an explicit mathematical expression for the efficiency of square foam blocks attached on center to circular tubes or HPs. The development involves extension of the validated numerical model, and proceeds as follows.

The ratio $\eta_{s q} / \eta_{a}$, is shown in Fig. 6(a) for various $r_{2} / r_{1}$ (or $L_{s q} /\left(2 r_{1}\right)$ ) over the range $0.001 \leq$ $m L_{c} \leq 2.5$. As expected from Fig. $5, \eta_{s q} / \eta_{a} \rightarrow 1$ with both efficiencies approaching unity as $m L_{c}$ $\rightarrow 0$. Discrepancies between $\eta_{s q}$ and $\eta_{a}$ increase as the difference in volumes of the annular and square porous blocks increase (as $r_{2} / r_{1}$ decreases). This effect is most prevalent at small values of $r_{2} / r_{1}$; for the $r_{2} / r_{1}=L_{s q} /\left(2 r_{1}\right)=1.2$ case, the square block has $89 \%$ more volume than the annular block (and $\eta_{s q} / \eta_{a} \sim 0.5$ at large $m L_{c}$ ). In contrast, for $r_{2} / r_{1}=10$ the square block has $28 \%$ more foam than the annular block (and $\eta_{s q} / \eta_{a} \sim 0.8$ at large $m L_{c}$ ).

With the effect of the foam geometry in mind, volume-weighted efficiencies $(\eta V)$ were also calculated and are reported in Fig. 6(b). In contrast to the dependence of $\eta_{s q} / \eta_{a}$ on $m L_{c}$ noted in Fig. 6(a), the ratio $\left(\eta_{s q} V_{s q}\right) /\left(\eta_{a} V_{a}\right) \rightarrow 1$ as $m L_{c} \rightarrow \infty$, due to the diminishing involvement of the outermost foam regions in the overall heat transfer process, as the length or diameter of the porous block becomes large. This collapse of volume-weighted efficiencies to similar values for $m L_{c} \gtrsim 2.5$ is consistent with infinite fin behavior exhibited for $m L_{c} \gtrsim 2.5$ for traditional fins [21].

Since $\eta_{s q} / \eta_{a} \rightarrow 1$ at small $m L_{c}$, and $\left(\eta_{s q} V_{s q}\right) /\left(\eta_{a} V_{a}\right) \rightarrow 1$ at large $m L_{c}$, analytical expressions for the annular porous block may be applied to the square block to determine the square block efficiency at small and large $m L_{c}$ using $\eta_{s q} / \eta_{a}$ and $\left(\eta_{s q} V_{s q}\right) /\left(\eta_{a} V_{a}\right)$, respectively. However, a generalized correlation(s), valid over the entire range of $m L_{c}$ is desired and is developed as follows. 
Use of a function that employs $K_{0}\left(m L_{c}\right)$ (defined in Eq. (19) and shown in Fig. 6(c)) appropriately weights the desirable behavior of Fig. 6(a) evident at small $m L_{c}$, with that of Fig. 6(b) at large $m L_{c}$

$$
f\left(m L_{c}\right) \equiv K_{0}\left(m L_{c}\right) \frac{\eta_{s q}}{\eta_{a}}+\left(1-K_{0}\left(m L_{c}\right)\right) \frac{\left(\eta_{s q} V_{s q}\right)}{\left(\eta_{a} V_{a}\right)} \square 1-e^{-2 \pi m L_{c}}
$$

and ultimately yields Eq. (20), which can be used to predict $\eta_{s q}$ solely as a function of $m L_{c}$ and $\eta_{a}$ (as given in Eq. (5)).

$$
\eta_{s q}=\frac{1-e^{-2 \pi m L_{c}}}{\frac{K_{0}\left(m L_{c}\right)}{\eta_{a}}+\left(1-K_{0}\left(m L_{c}\right)\right) \frac{V_{s q}}{\left(\eta_{a} V_{a}\right)}}
$$

Square porous block efficiencies determined with $(i)$ the analytically verified numerical model along with Eq. (19) and (ii) Eq. (20) are reported in Fig. 6(d). Overall, the agreement between the numerically- and analytically determined efficiencies is good, with the larger discrepancies occurring at the smaller values of $m L_{c}$ and $L_{s q} /\left(2 r_{1}\right)$ (as can be seen upon close inspection of Fig. 6(d). However, it should be noted that there exists a value of $L_{s q} /\left(2 r_{1}\right)$ below which a single pore would occupy the entire lateral block dimension and use of either the analysis or numerical model of this study would be inappropriate. For the square foam and value of $L_{s q}$ used here, the limit is $L_{s q} q\left(2 r_{1}\right)_{\text {, min }}=\left(L_{s q} / 2+D_{p}\right) / r_{1}=1.2$. In addition, since the efficiencies for either the annular or square foam are near unity at small values of $m L_{c}$, as evident in Figs. 5(b -d), Eq. (5) performs better than Eq. (20) in predicting the efficiency of the square foam block.

The recommended analytical expressions to determine the porous matrix efficiencies of both annular and square blocks are listed in Table 1. Also shown are the recommended ranges of application as well as the average and maximum discrepancies between the analytical values and 
numerical predictions. Note that although discrepancies are reported for a limited range of $m L_{c}$, the analytical expressions may be applied to any value of $m L_{c}$ as evident in Fig. 6(c).

\subsection{Comparison of predicted and measured heat transfer rates}

Equation (20) is now employed to predict the value of $R_{f}$ and the experimentally measured heat transfer rates. Experiments were performed with four ambient velocities $\left(u_{\infty}=\right.$ $0.18,0.47,0.86$, and $1.16 \mathrm{~m} / \mathrm{s})$ for $L_{s q} /\left(2 r_{1}\right)=3.9$. At each velocity three excess temperatures, $\theta=$ $T_{t c}-T_{\infty} \approx 20,35$, and $45^{\circ} \mathrm{C}$, were investigated experimentally.

An appreciable increase in the air temperature (defined here to be a maximum increase in its temperature relative to $\theta_{b}$ exceeding $5 \%$ ) is anticipated for the experimental flow rates and thermal conditions used here, necessitating employment of a log mean temperature difference (LMTD) across the foam resistance of the thermal resistance network of Fig. 3(c)

$$
q=\frac{\mathrm{LMTD}}{R_{f, s q}}
$$

where

$$
\mathrm{LMTD}=T_{b}-T_{l m}=\frac{\left(T_{b}-T_{\infty}\right)-\left(T_{b}-T_{f, o}\right)}{\ln \left(\frac{T_{b}-T_{\infty}}{T_{b}-T_{f, o}}\right)}=\frac{T_{f, o}-T_{\infty}}{\ln \left(\frac{T_{b}-T_{\infty}}{T_{b}-T_{f, o}}\right)}
$$

and

$$
R_{f, s q}=\frac{1}{\alpha_{v}\left(L_{s q}^{2}-\pi r_{1}^{2}\right) t \eta_{s q} h}
$$

where $T_{f, o}$ is the average air temperature at the outlet of the foam. Thermal energy passing from the heat spreader, at $T_{t c}$, to the base of the foam is 


$$
q=\frac{T_{t c}-T_{b}}{R_{\mathrm{tot}-f, s q}}
$$

and

$$
q=\dot{m} c_{p, f}\left(T_{f, o}-T_{\infty}\right)
$$

Equations (21) through (25) may be combined to yield

$$
q=\left(T_{t c}-T_{\infty}\right) \frac{1-e^{\frac{1}{\dot{m} c_{p, f} R_{f, s q}}}}{R_{\mathrm{tot}-f, s q}-\left(R_{\mathrm{tot}-f}+\frac{1}{\dot{m} c_{p, f}}\right) e^{\frac{1}{\dot{m} c_{p, f} R_{f, s q}}}}
$$

where $\dot{m}$ is the mass flow rate of air and air properties are evaluated at $T=\left(T_{b}+T_{l m}\right) / 2$.

Predicted heat rates. Predicted and measured heat transfer rates are reported in Fig. 7. Note that the uncertainty in the heat rates is $\pm 3 \%$ of the reported values, while the uncertainty in the reported values of the measured excess temperatures is $\pm 1 \%$.

The agreement between the predicted and measured heat rates is considered to be excellent. The values of $R_{f, s q}$, determined using Eq. (23), are $0.72,0.58,0.48$, and $0.43 \mathrm{~K} / \mathrm{W}$ for the $u_{\infty}=0.18,0.47,0.86$, and $1.16 \mathrm{~m} / \mathrm{s}$ cases, respectively.

Experimentally inferred porous matrix resistances. Measured values of $R_{f, s q}$ may be deduced from the experimentally determined heat rates and temperatures in conjunction with the thermal resistance network of Fig. 3(c). The values $R_{f, s q}$ are $0.74 \pm 0.04,0.62 \pm 0.03,0.53 \pm 0.02$, and $0.41 \pm 0.01 \mathrm{~K} / \mathrm{W}$ for the $u_{\infty}=0.18,0.47,0.86$, and $1.16 \mathrm{~m} / \mathrm{s}$ cases, respectively, reflecting an average difference of approximately $6 \%$ from the values determined from Eq. (23) reported above. 
It should be noted that the preceding predictions of $q$ and the inferred values of $R_{f, s q}$ depend on the values of the remaining thermal resistances in the network of Fig. 3(c). The resistance with the largest uncertainty is that of the contact between the insert and the foam. Increasing (decreasing) $R_{t, c}^{\prime \prime}$ by $10 \%$ decreases (increases) predicted values of $q$ by approximately $3 \%$ and decreases (increases) $R_{f, s q}$ by approximately $7 \%$.

\section{Conclusions and Recommendations}

Novel, analytical expressions have been developed for the thermal efficiency and thermal resistance associated with convection and conduction within an open-cell metal foam (of cylindrical shape) that is attached to a circular tube. Using a two-temperature porous medium numerical model (validated by comparing its predictions to those of the analytical expression), a companion semi-analytical expression for the thermal efficiency of square-shaped, open-cell metal foam blocks attached on-center to a circular tube (Eq. (20)) is also developed. To demonstrate its usage, the semi-analytical model for square foam blocks is employed to predict experimentally measured heat transfer rates, as well as experimentally inferred values of the foam thermal resistances in a unique experimental setup. The new analytical and semi-analytical expressions for the thermal efficiency of both annular and square-shaped foams are summarized

in Table 1, along with their broad recommended ranges of application and levels of uncertainty.

Because the forms of the governing one-dimensional heat equation and boundary conditions for the annular foams considered here are identical to those of a convectively heated or cooled thin, solid annular fin with an adiabatic tip [21], the extension methodology developed in this study may also be used to extend well-known expressions for the thermal efficiency of an annular thin fin [21] to thin fins of square shapes attached on-center to cylindrical tubes. As 
implied in the discussion of the experimental apparatus and experimental results, novel approaches are desirable to both more accurately quantify and minimize thermal contact resistances at the foam-tube interface.

\section{Acknowledgments}

This material is based upon work supported by the National Science Foundation under Grant Nos. 1435131 (University of Kansas) and 1435233 (University of Connecticut). The authors also wish to thank Will Libeer and Trey Fox, as well as Dr. Nourouddin Sharifi, for their assistance in the construction and validation of the experimental apparatus.

\section{References}

[1] C.Y. Zhao, Review on thermal transport in high porosity cellular metal foams with open cells, International Journal of Heat and Mass Transfer 55 (2012) 3618-3632.

[2] I. Ghosh, How good is open-cell metal foam as a heat transfer surface, ASME Journal of Heat Transfer 131 (2009) 101004-1-8.

[3] L. Tadrist, M. Miscevic, O. Rahli, F. Topin, About the use of fibrous materials in compact heat exchangers, Experimental Thermal and Fluid Science 28 (2004) 193-199.

[4] D.L. Koch, R.G. Cox, H. Brenner, J.F. Brady, The effect of order on dispersion in porous media, Journal of Fluid Mechanics 200 (1989) 173-188.

[5] T.J. Lu, H.A. Stone, M.F. Ashby, Heat transfer in open-cell metal foams, Acta Materialia 46 (10) (1998) 3619-3635.

[6] P. Du Plessis, A. Montillet, J. Comiti, J. Legrand, Pressure drop prediction for flow through high porosity metallic foams, Chemical Engineering Science 49 (21) (1994) 3545-3553.

[7] D. Weaire, R. Phelan, A counter-example to Kelvin's conjecture on minimal surfaces, Philosophical Magazine Letters 69 (1994) 107-110. 
[8] K. Boomsma, D. Poulikakos, F. Zwick, Metal foams as compact high performance heat exchangers, Mechanics of Materials 35 (12) (2003) 1161-1176.

[9] A. Kopanidis, A. Theodorakakos, E. Gavaises, D. Bouris, 3D numerical simulation of flow and conjugate heat transfer through a pore scale model of high porosity open cell metal foam, International Journal of Heat and Mass Transfer 53 (11-12) (2010) 25392550.

[10] S. Krishnan, J.Y. Murthy, S.V. Garimella, Direct simulation of transport in open-cell metal foams, ASME Journal of Heat Transfer 128 (2006) 793-799.

[11] Lord Kelvin (Sir William Thomson), On the division of space with minimum partitional area, Philosophical Magazine 24 (151) (1887) 121-134.

[12] M. Iasiello, S. Cunsolo, M. Oliviero, W.M. Harris, N. Bianco, W.S.K. Chiu, V. Naso, Numerical analysis of heat transfer and pressure drop in metal foams for different morphological models, ASME Journal of Heat Transfer 136 (11) (2014) 112601:1-10.

[13] P. Ranut, E. Nobile, L. Mancini, Microtomography-based CFD analysis of transport in open-cell aluminum metal foams, Journal of Physics: Conference Series 501 (1) (2014) $1-11$.

[14] D.Y. Lee, K. Vafai, Analytical characterization and conceptual assessment of solid and fluid temperature differentials in porous media, International Journal of Heat and Mass Transfer 42 (3) (1999) 423-435.

[15] V.V. Calmidi, R.L. Mahajan R.L., Forced convection in high porosity metal foams, ASME Journal of Heat Transfer 122 (3) (2000) 557-565.

[16] T. Dixit, I. Ghosh, An experimental study on open cell metal foam as extended heat transfer surface, Experimental Thermal and Fluid Science 77 (2016) 28-37.

[17] I. Ghosh, Heat transfer correlation for high-porosity open-cell foam, International Journal of Heat and Mass Transfer 52 (5-6) (2009) 1488-1494.

[18] S.H. Park, T.H. Kim, J.H. Jeong, Experimental investigation of the convective heat transfer coefficient for open-cell porous metal fins at low Reynolds numbers, International Journal of Heat and Mass Transfer 100 (2016) 608-614.

[19] P. De Jaeger, C. T'Joen, H. Huisseune, B. Ameel, S. De Schampheleire, M. De Paepe, Assessing the influence of four cutting methods on the thermal contact resistance of 
open-cell aluminum foam, International Journal of Heat and Mass Transfer 55 (21-22) (2012) 6142-6151.

[20] T.M. Jeng, S.C. Tzeng, Y.H. Hung, An analytical study of local thermal equilibrium in porous heat sinks using fin theory, International Journal of Heat and Mass Transfer (49) (11-12) (2006) 1907-1914.

[21] T.L. Bergman, A.S. Lavine, F.P. Incropera, D.P. Dewitt, Fundamentals of Heat and Mass Transfer, Seventh ed., Wiley, Hoboken, 2011.

[22] C.Y. Zhao, W. Lu, S.A. Tassou, Thermal analysis on metal-foam filled heat exchangers. Part II: Tube heat exchangers, International Journal of Heat and Mass Transfer 49 (1516) (2006) 2762-2770.

[23] C. T'Joen, P. De Jaeger, H. Huisseune, S. Van Herzeele, N. Vorst, M. De Paepe, Thermo-hydraulic study of a single row heat exchanger consisting of metal foam covered round tubes, International Journal of Heat and Mass Transfer 53 (15-16) (2010) 32623274.

[24] M. Odabee, K. Hooman, H. Gurgenci, Metal foam heat exchangers for heat transfer augmentation from a cylinder in cross-flow, Transport in Porous Media 86 (3) (2011) 911-923.

[25] S.P. Benn, L.M. Poplaski, A. Faghri, T.L. Bergman, Analysis of thermosyphon/heat pipe integration of dry cooling for thermoelectric power generation, Applied Thermal Engineering 104 (2016) 358-374.

[26] H. Shabgard, T.L. Bergman, N. Sharifi, A. Faghri, High temperature latent heat thermal energy storage using heat pipes, International Journal of Heat and Mass Transfer 53 (1516) (2010) 2979-2988.

[27] H. Shabgard, M.J. Allen, N. Sharifi, S.P. Benn, A. Faghri, T.L. Bergman, Heat pipe heat exchangers and heat sinks: Opportunities, challenges, applications, analysis, and state of the art, International Journal of Heat and Mass Transfer 89 (2015) 138-158.

[28] V.V. Calamidi, Transport Phenomenon in High Porosity Fibrous Metal Foams, PhD thesis, University of Colorado, Boulder, CO, 1997.

[29] F. Wang, X. Li, The stagnant thermal conductivity of porous media predicted by the random walk theory, International Journal of Heat and Mass Transfer 107 (2017) 520533. 
[30] V.V. Calmidi, R.L. Mahajan, The effective thermal conductivity of high porosity fibrous metal foams, ASME Journal of Heat Transfer 121 (2) (1999) 466-471.

[31] K. Vafai, C.L. Tien, Boundary and inertia effects on flow and heat transfer in porous media, International Journal of Heat and Mass Transfer 24 (2) (1981) 195-203.

[32] K. Vafai, S.J. Kim, Forced convection in a channel filled with a porous medium: an exact solution, ASME Journal of Heat Transfer 111 (4) (1989) 1103-1106.

[33] J.R. Stark, N. Sharifi, T.L. Bergman, A. Faghri, An experimentally verified numerical model of finned heat pipes in crossflow, International Journal of Heat and Mass Transfer 97 (2016) 45-55.

[34] N. Sharifi, T.L. Bergman, M.J. Allen, A. Faghri, Melting and solidification enhancement using a combined heat pipe, foil approach, International Journal of Heat and Mass Transfer 78 (2014) 930-941.

[35] N. Sharifi, J.R. Stark, T.L. Bergman, A. Faghri, The influence of thermal contact resistance on the relative performance of heat pipe-fin array systems, Applied Thermal Engineering 105 (2016) 46-55.

[36] E. Oberg, Machinery's Handbook, $29^{\text {th }}$ ed., Industrial Press, New York, 2012, 18151867.

[37] E. Sadeghi, S. Hsieh, M. Bahrami, Thermal conductivity and contact resistance of metal foams, Journal of Physics D: Applied Physics 44 (12) (2011) 125406:1-7.

[38] Metals Handbook, 10th Ed., v.2, ASM Handbook Committee, American Society for Metals, USA, 1990. 


\section{Appendix. Grid independence}

Simulations for the square and annular porous media geometries, as outlined in Section 3, were investigated for their grid dependence for the $r_{2} / r_{1}=L_{s q} /\left(2 r_{1}\right)=1.5$ and 5 , and 10 geometries and the $m L_{c}=0.1$ and 2 cases. The grid dependence study discussed here (corresponding to $r_{2} / r_{1}=L_{s q} /\left(2 r_{1}\right)=10$ and $\left.m L_{c}=2\right)$ is representative of all geometries and cases. Results of the predicted efficiency, the volumetrically averaged temperature of the porous matrix, and the heat rate were compared for computational grids ranging from 560 (228) to 1.80 million (1.78 million) nodes for the annular (square) geometries. Convergence was assessed by normalizing the monitored quantities to results corresponding to the highest node count. Predictions for both the annular and square geometries were within $0.1 \%$ of the highest resolution results for grids greater than approximately 1200 nodes (e.g., predictions of the porous matrix efficiency are shown in Fig. A-1). Grids consisting of of 110,960 and 122,056 nodes were employed for $r_{2} / r_{1}=L_{s q} /\left(2 r_{1}\right)=10$, which produced results within $0.01 \%$ of those associated with the highest resolution grid. Similar grids were employed for all geometries and cases. 
Table 1. Recommended expressions for the porous matrix efficiencies of annular and square porous blocks; $1.5 \leq r_{2} / r_{1}=L_{s q} /\left(2 r_{1}\right) \leq 10$.

\begin{tabular}{|c|c|c|c|c|}
\hline & Recommended Expressions & \begin{tabular}{|c} 
Recommended \\
Range
\end{tabular} & $\begin{array}{l}\text { Avg. Error } \\
\text { (Max. Error) }\end{array}$ & Eq. \\
\hline 离 & $\eta_{a}=\frac{2 r_{1}}{m\left(r_{2}^{2}-r_{1}^{2}\right)}\left(\frac{K_{1}\left(m r_{1}\right) I_{1}\left(m r_{2}\right)-I_{1}\left(m r_{1}\right) K_{1}\left(m r_{2}\right)}{K_{0}\left(m r_{1}\right) I_{1}\left(m r_{2}\right)+I_{0}\left(m r_{1}\right) K_{1}\left(m r_{2}\right)}\right)$ & $\begin{array}{c}\quad 0 \leq m L_{c} \leq \infty \\
\text { where } L_{c}=r_{2}-r_{1}\end{array}$ & $\begin{array}{c}0.05 \% \\
(0.59 \%) \\
\quad \text { for } \\
0 \leq m L_{c} \leq 2.5\end{array}$ & $(5)$ \\
\hline \multirow{2}{*}{  } & $\eta_{s q} \approx \frac{2 r_{1}}{m\left(\left(L_{s q} / 2\right)^{2}-r_{1}^{2}\right)}\left(\frac{K_{1}\left(m r_{1}\right) I_{1}\left(m L_{s q} / 2\right)-I_{1}\left(m r_{1}\right) K_{1}\left(m L_{s q} / 2\right)}{K_{0}\left(m r_{1}\right) I_{1}\left(m L_{s q} / 2\right)+I_{0}\left(m r_{1}\right) K_{1}\left(m L_{s q} / 2\right)}\right)$ & $\begin{aligned} 0 \leq m L_{c} & \leq 0.4 \\
\text { where } L_{c} & =\frac{L_{s q}}{2}-r_{1}\end{aligned}$ & $\begin{array}{c}0.48 \% \\
\quad(4.7 \%) \\
\quad \text { for } \\
0 \leq m L_{c} \leq 0.4\end{array}$ & $(5)$ \\
\hline & $\eta_{s q}=\frac{1-e^{-2 \pi m L_{s q}}}{\frac{K_{0}\left(m L_{s q}\right)}{\eta_{a}}+\left(1-K_{0}\left(m L_{s q}\right)\right) \frac{V_{s q}}{\left(\eta_{a} V_{a}\right)}}$ & $\begin{array}{c}\quad 0.4<m L_{c} \leq \infty \\
\text { where } L_{c}=\frac{L_{s q}}{2}-r_{1}\end{array}$ & $\begin{array}{c}0.74 \% \\
(4.7 \%) \\
\quad \text { for } \\
0.4 \leq m L_{c} \leq 2.5\end{array}$ & (20) \\
\hline
\end{tabular}




\section{Figure captions}

Fig. 1. Photograph of 10 PPI aluminum Duocel® foam.

Fig. 2. Description of the 1D annular analytical domain. (a) overall domain, (b) annular control volume energy balance.

Fig. 3. Numerical domains for the (i) annular and (ii) square geometries.

Fig. 4. Experimental setup. (a) centerline view of the channel and heat pipe carrier, (b) detail of the heat pipe and foam, (c) thermal resistance network.

Fig. 5. Analytically- and numerically predicted porous matrix efficiencies versus $m L_{c}$. (a) annular analytical and numerical predictions for $r_{2} / r_{1}=L_{s q} /\left(2 r_{1}\right)=1.2,1.5,2,3,5,7.5$, and 10, (b) predicted efficiencies for $r_{2} / r_{1}=L_{s q} /\left(2 r_{1}\right)=2$, (c) predicted efficiencies for $r_{2} / r_{1}=5$, (d) predicted efficiencies for $r_{2} / r_{1}=L_{s q} /\left(2 r_{1}\right)=10$.

Fig. 6. Relationship of analytically- and numerically predicted porous matrix efficiencies versus $m L_{c}$. (a) ratio of square to annular efficiencies, (b) ratio of square to annular volume-weighted efficiencies, (c) scaled Bessel function from Eq. (20) for various $r_{2} / r_{1}=L_{s q} /\left(2 r_{1}\right)$, (d) square semi-analytical and numerical predictions for $L_{s q} /\left(2 r_{1}\right)=1.2,1.5,2,3,5,7.5$, and 10 .

Fig. 7. Experimentally measured and semi-analytical predicted heat rates for a square porous matrix under various ambient velocity and thermal conditions.

Fig. A-1. Predicted efficiencies versus mesh count. 


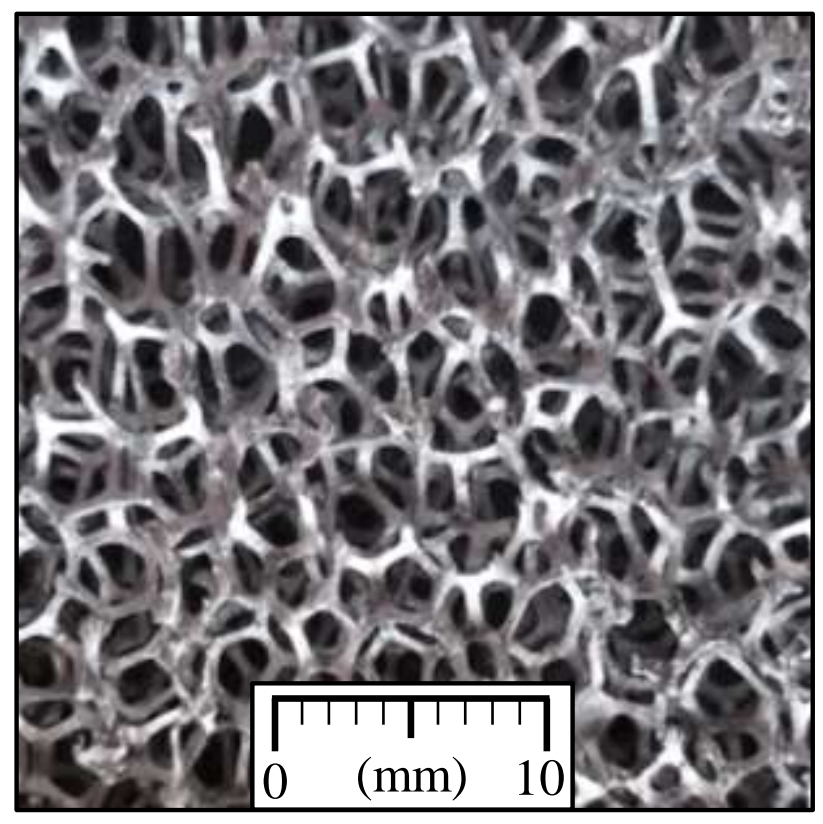

Fig. 1 


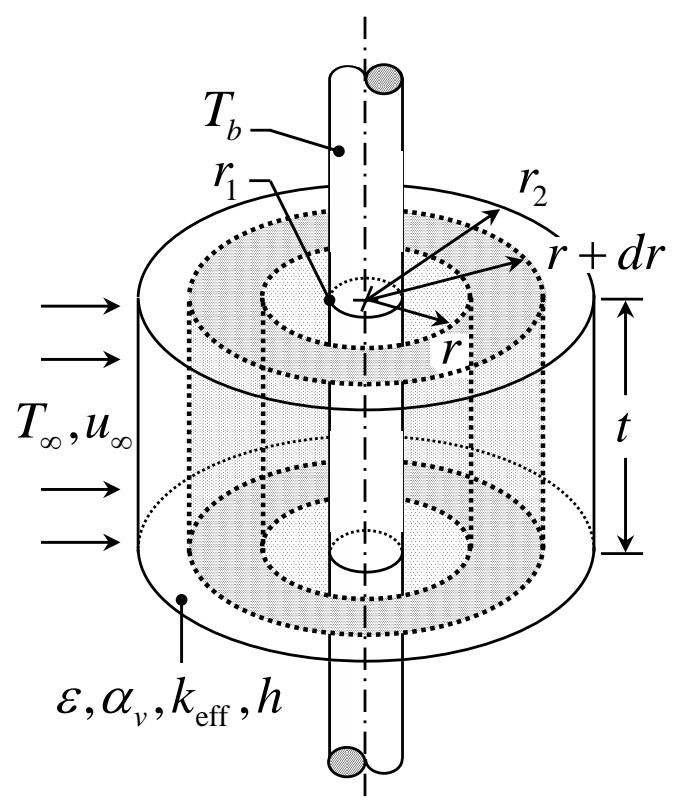

(a)

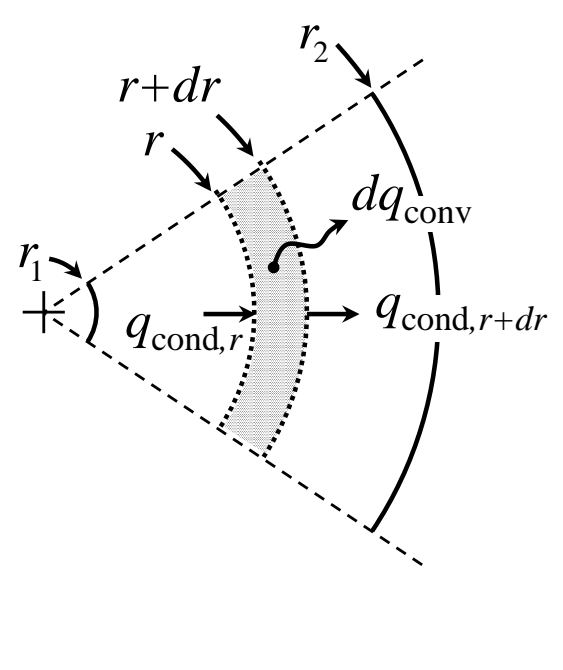

(b)

Fig. 2 


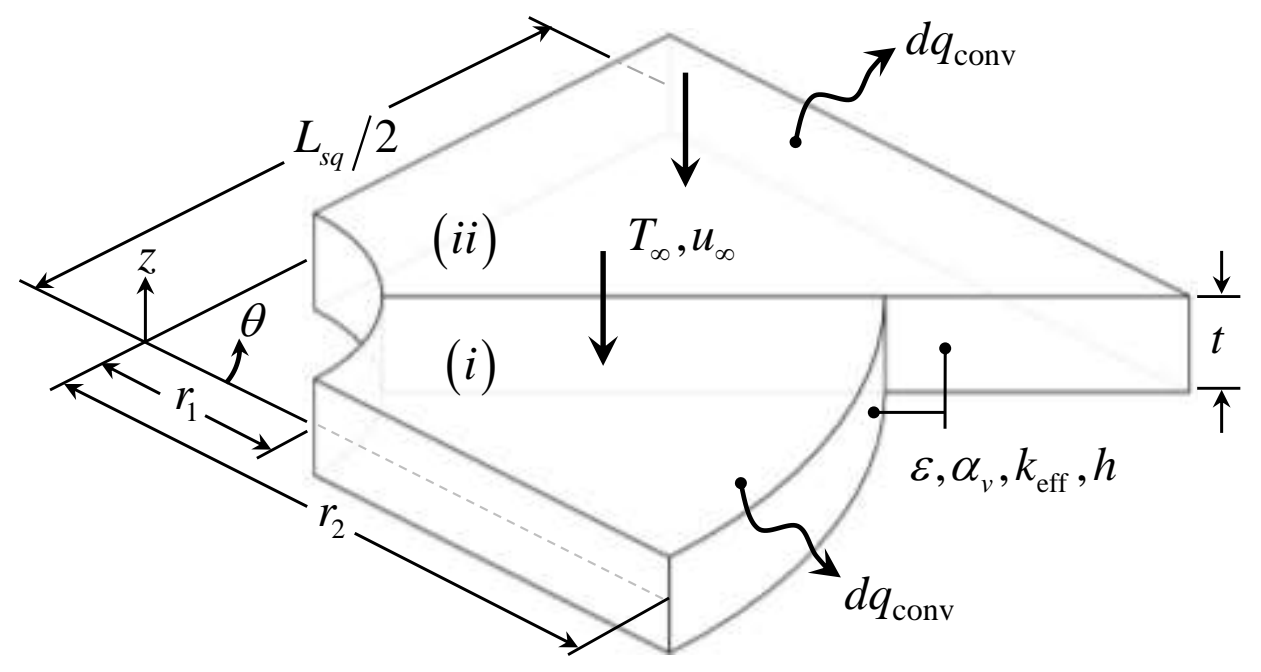

Fig. 3 


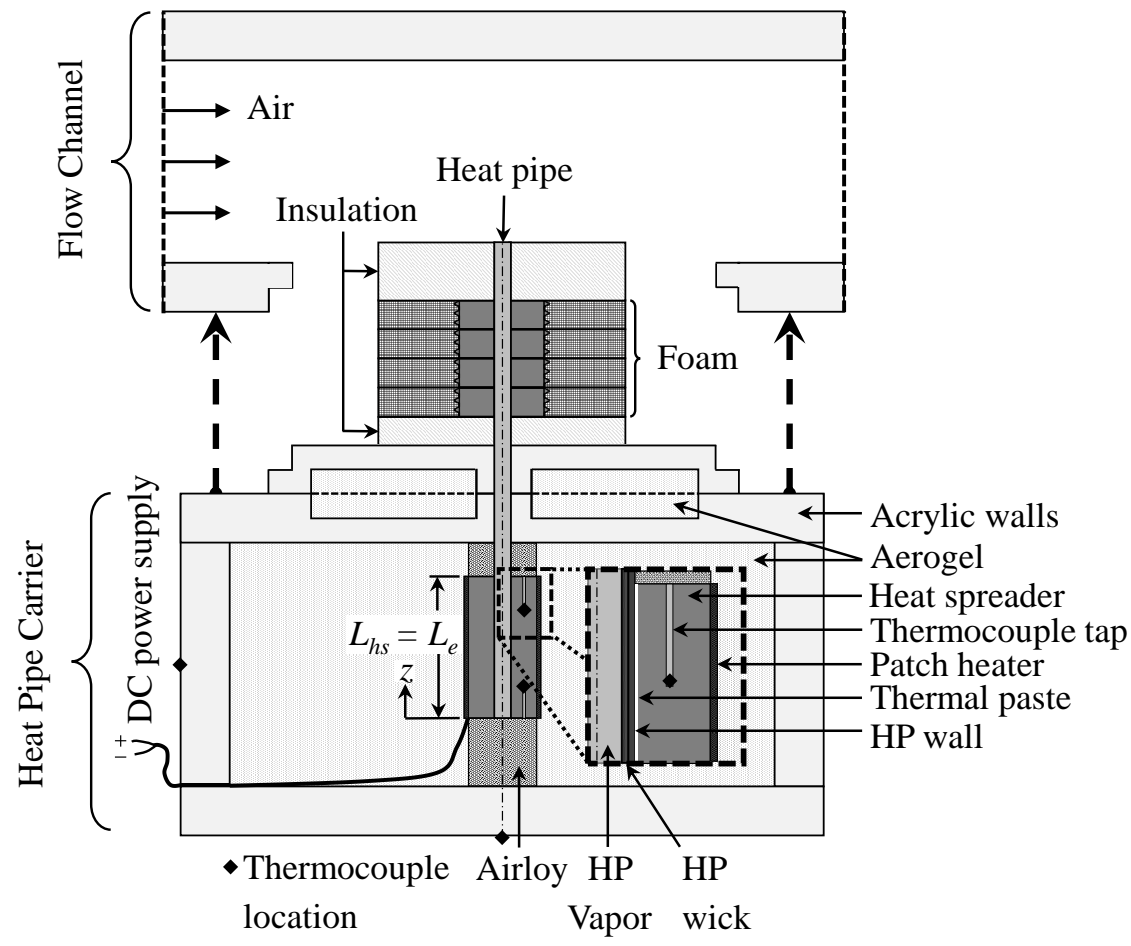

(a)



Fig. 4 

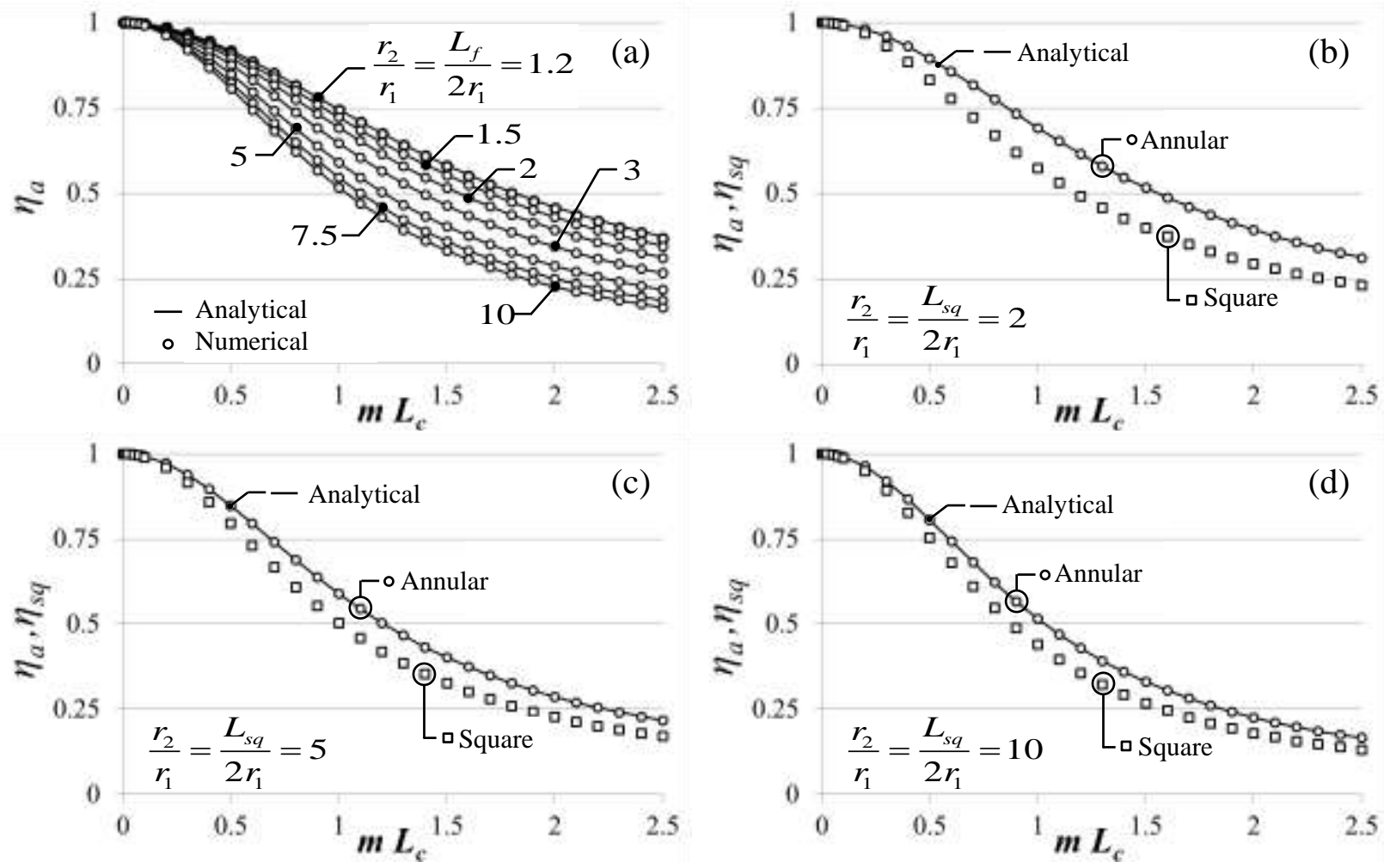

Fig. 5 



Fig. 6 


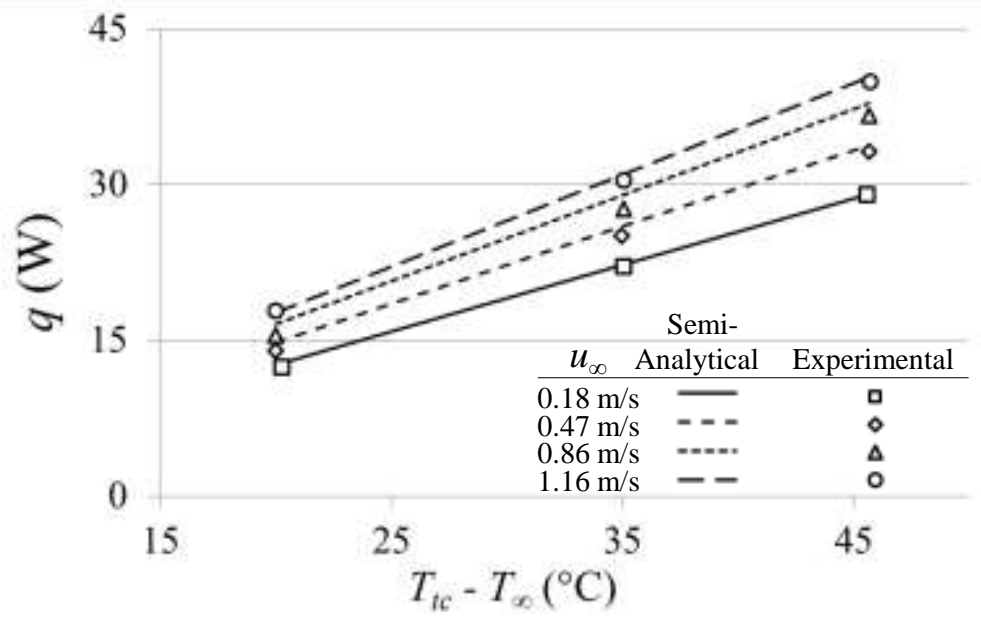

Fig. 7 


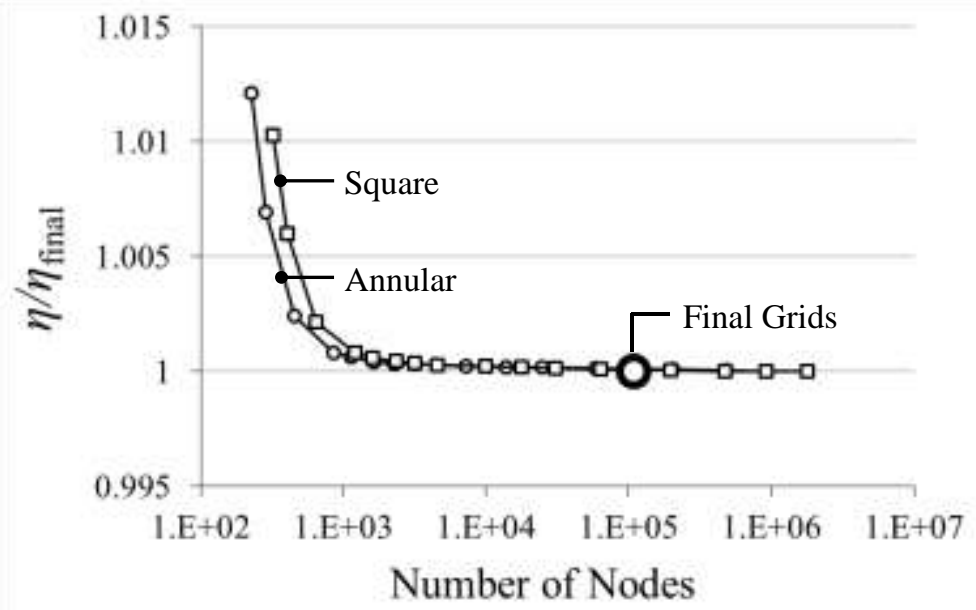

Fig. A-1 